Selective medium for isolating Arcanobacterium haemolyticum

Arcanobacterium haemolyticum is a facultatively anaerobic Gram positive bacillus previously known as Corynebacterium haemolyticum. It is most commonly isolated from the upper respiratory tract of patients with pharyngitis, ${ }^{12}$ but has also been isolated from skin lesions and occasionally from systemic infections. Isolation of $A$ haemolyticum from healthy subjects is rare. ${ }^{2}$

The only medium previously described for the isolation of $A$ haemolyticum is an enriched agar containing human blood or horse blood. After 48 hours of incubation on this medium $A$ haemolyticum produces colonies which characteristically, have a central pit and are surrounded by a zone of complete haemolysis. Despite the use of enriched media, isolation of $A$ haemolyticum can be difficult as the organism is slow growing and is easily masked by commensal flora. The organism may therefore be a more common cause of pharyngitis than is currently recognised. We have developed a selective medium suitable for its isolation.

In a study of the antimicrobial susceptibilities of $A$ haemolyticum we found that all 26 strains examined were resistant to mupirocin (minimum inhibitory concentrations $>128 \mathrm{mg} / \mathrm{l}$ ). Mupirocin is highly active against commensal staphylococci and streptococci. ${ }^{4}$ Aztreonam and amphotericin $B$ were used to inhibit the growth of Gram negative bacteria and yeasts, respectively. The complete medium consisted of a blood agar base Oxoid No 2) containing 5\% horse biood, 8 $\mathrm{mg} / \mathrm{l}$ mupirocin (Beecham), $4 \mathrm{mg} / 1$ aztreonam (Squibb) and $1 \mathrm{mg} / \mathrm{l}$ amphotericin B (Sigma)

Strains from the National Collection of Type Cultures and clinical isolates of $A$ haemolyticum grew well on the selective medium and produced characteristic colonies with narrow zones of complete haemolysis and a central pit.

The efficacy of this medium for the isolation of $A$ haemolyticum from clinical specimens was investigated during February and March 1989. All throat swabs received by Chelmsford Public Health Laboratory were inoculated on to the selective medium and on to conventional horse blood agar. The inoculated media were incubated for 48 hours at $37^{\circ} \mathrm{C}$ in an anaerobic atmosphere contain-

Isolation of Arcanobacterium haemolyticum from throat swabs of patients in various age ranges

\begin{tabular}{llll}
\hline & & \multicolumn{2}{l}{ Number of isolates } \\
\cline { 3 - 4 } Age range (years) & Number of specimens & Blood agar & Selective medium \\
\hline $0-10$ & 215 & 0 & 0 \\
$11-20$ & 126 & 2 & 8 \\
$21-30$ & 92 & 0 & 1 \\
$>31$ & 175 & 0 & 0 \\
Unknown & 65 & 2 & 9 \\
Total & 673 & 0 & 9 \\
\hline
\end{tabular}

Colorimetric determination of human albumin

Many methods, based on a variety of principles, have been described for the measurement of human albumin. Increases in serum albumin are almost exclusively due to dehydration. Decreases are seen in patients with (i) excessive protein loss due to kidney damage or severe haemorrhage or burns, (ii) impaired or genetic disorders, (iii) inadequate ing $10 \%$ carbon dioxide. Both media were examined for characteristic colonies of haemolyticum. Identification was confirmed biochemically.

A haemolyticum was isolated from nine of 673 specimens (table). Isolation was much better with the selective medium as only two of the nine isolates were detected on conventional blood agar. The selective medium greatly reduced the growth of commensal organisms, thus permitting easier recognition of $A$ haemolyticum. In the group aged 11-20 years the organism was isolated from eigh $(6.3 \%)$ of the 126 specimens. A similar specific age association has been noted by others. $^{2}$ The selective medium would therefore be of most value for the culture of throat swabs from teenagers or young adults. Lancefield group A streptococci were isolated from $18(14.3 \%)$ of the 126 specimens from patients aged 11-20 years, so $A$ haemolyticum seems to be a relatively important pathogen in this age group.

Erythromycin is the antibiotic of choice for treatment. ${ }^{1}$ With the recent concern over erythromycin resistance in Lancefield group A streptococci, ${ }^{5}$ however, it may not be the initial choice for the empirical treatment of pharyngitis. Precise identification of the infecting organism would seem desirable. Use of a selective medium such as that described would clearly facilitate recognition. NP BRENWALD Clinical Microbiology and Public Health Laboratory Addenbrookes Hospital Cambridge CB2 $2 Q W$ EL TEARE

LK MOUNTFOR RE TETTMAR Public Health Laboratory,

Chelmsford CM2 OYX

1 Selander B, Ljungh A. Corynebacterium haemolyticum as a cause of nonstreptococcal pharyngitis. J Infect Dis 1986;154:1041.

2 Banck G, Nyman $M$. Tonsillitis and rash associated with Corynebacterium haemolyticum. J Infect Dis 1986;154:1037-40.

3 Clarridge JE. The recognition and significance of Arcanobacterium haemolyticum. Clin Microbiol 1989;11:41-5.

4 Sutherland R, Boon RJ, Griffin LE, et al. Antibacterial activity of Mupirocin (Pseudomonic acid), a new antibiotic for topical use. Antimicrob Agents Chemother 1985;27:495-8.

5 Jarvinen $\mathrm{H}$, Nissinen A, Huovinen $\mathrm{P}$, et al. Erythomycin resistance in group $\mathbf{A}$ streptococci. Lancet 1989;i:1022-3.

\section{Number of isolates}

protein intake seen in malnutrition and (iv) gastrointestinal disorders with malabsorption, vomiting, or diarrhoea (before dehydration)

Methods for the quantitative measurement of serum or plasma albumin fall into four categories-namely, salt fractionation, electrophoresis, dye-binding and immunochemical. Dye-binding techniques are currently the most widely used in routine clinical chemistry as they are readily automated, inexpensive, simple and give reproducible results. Data from the United Kingdom External Quality Assessment Scheme for General Clinical Chemistry indicated that $98 \%$ of participating laboratories measure serum albumin by either bromocresol green (BCG) or bromocresol purple (BCP) dye-binding methods; of these, BCG methods remain the most widely used. A lack of specificity of BCG for albumin, however, has led the International Federation of Clinical Chemistry Expert Panel on Proteins ${ }^{1}$ and other authors ${ }^{2-4}$ to recommend that the method should only be used for screening purposes. It has been reported that not only does BCG overestimate low albumin concentrations, but that it also underestimates concentrations in the high normal range. ${ }^{2}$

The following assay (patent-pending) uses the specificity of the reduction of BSPT (2(2'-benzothiazolyl)-5-styrl-3-pthal-hydrazidyl)-tetrazolium chloride to its coloured formazan, in the presence of a reducing agent, electron carrier, and human albumin and benefits from simple colorimetric detection.

To $50 \mathrm{ul}$ serum add $1 \mathrm{ml}$ buffered colour reagent containing BSPT, methoxy $-\mathrm{N}$ methyl phenazinium methyl sulphate, and dithio-threitol. After incubation for two minutes at room temperature the absorbance is read at $590 \mathrm{~nm}$

The procedure is linear over the albumin range $1-80 \mathrm{mg} / \mathrm{ml}$. No interference was found with transferrin, bilirubin, or heparin and the method compared well with traditional dyebinding and immunological assays.

RJ HINTON BS MALLON J MIIRIS T ATKINSON PM HAMMOND Diagnostic Enzymology Group, Division of Biotechnology

PHLS Centre for Applied Microbiology and
Research,
Porton Down, Salisbury.
CP PRICE

PHLS Centre for Applied Microbiology and
Research,
Porton Down, Salisbury.
CP PRICE

PHLS Centre for Applied Microbiology and
Research,
Porton Down, Salisbury.
CP PRICE Department of Clinical Biochemistry, Turner Street, London E1 $2 A D$
Tulan

1 International Federation of Clinical Chemistry. Committee on standards and expert panel news. Newsletter 1976;13:5.

2 Webster D, Bignell AHC, Attwood EC. An assessment of the suitability of bromocresol green for the determination of serum albumin. Clin Chim Acta 1984;53:101-8.

3 Ferreira P, Price CP. A comparison of bromocresol green and immunoprecipitation methods for the determination of serum albumin. Clin Chim Acta 1974;55:259-62.

4 Slater L, Carter PM, Hobbs JR. Measurement of albumin in the sera of patients. Ann Clin Biochem 1975;12:33-40.

\section{MATTERS ARISING}

Throat culture from patients with meningococcal meningitis

Cartwright and Jones suggest that throat culture can be useful in the diagnosis of meningococcal meningitis when the patients have taken antibiotics before admission into hospital. ${ }^{1}$ This approach seems valid in the 